\title{
Neuromorphic computing with integrated photonics and superconductors
}

\author{
Jeffrey M. Shainline, Sonia M. Buckley, Richard P. Mirin, and Sae Woo Nam \\ National Institute of Standards and Technology \\ 325 Broadway, Boulder, CO, USA \\ Email: jeffrey.shainline@nist.gov
}

\begin{abstract}
We present a hardware platform combining integrated photonics with superconducting electronics for large-scale neuromorphic computing. Semiconducting few-photon light-emitting diodes work in conjunction with superconducting-nanowire singlephoton detectors to behave as spiking neurons. These neurons are connected through a network of waveguides, and variable weights of connection can be implemented using several approaches. These processing units can operate at $20 \mathrm{MHz}$ with fully asynchronous activity, light-speed-limited latency, and power densities on the order of $1 \mathrm{~mW} / \mathrm{cm}^{2}$. The processing units achieve an energy efficiency of $20 \mathrm{aJ} /$ synapse event, an improvement of six orders of magnitude over recent CMOS demonstrations [1]. We present calculations showing this approach could scale to interconnectivity near that of the human brain, and could surpass the brain in speed and efficiency.
\end{abstract}

\section{INTRODUCTION}

As the lithographic scaling which has enabled Moore's law reaches fundamental limitations, new computational architectures are gaining attention. Guided by the efficiency and speed of biologically evolved computers when performing certain tasks, neuromorphic architectures are particularly intriguing. Such a platform requires individual processing units (neurons) which integrate signals from a large number of other neurons and produce an output pulse (action potential) when the integrated signals reach a certain threshold. The thresholds of each of the neurons as well as the strength of connection between the neurons must be dynamic and malleable based on system activity. For advanced neuromorphic systems, scaling to billions of inter-

U.S. Government work not protected by U.S. copyright

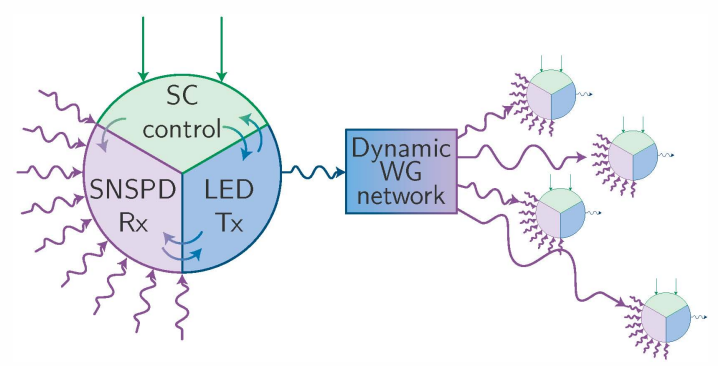

Fig. 1. Schematic representation of the proposed device concept. SC = superconducting; SNSPD = superconducting-nanowire single-photon detector; $\mathrm{Rx}=$ receive; $\mathrm{Tx}=$ transmit; $\mathrm{WG}=$ waveguide.

connected units is necessary. Such scaling requires each neuron to be compact and energy efficient.

Here we propose an optoelectronic hardware platform utilizing waveguide-integrated semiconductor light emitters with superconducting detectors and electronics to behave as spiking neurons. Optical signals are communicated through reconfigurable nanophotonic waveguides, capturing the interconnectivity of a biological neuron's dendritic arbor. Firing thresholds and neuron gain are controlled by a dynamic superconducting network which can be reconfigured with photonic signals. A schematic representation of the concept is shown in Fig. 1.

Utilization of light-emitting semiconductors allows access to photonic degrees of freedom (frequency, polarization, mode index, intensity, statistics, and coherence) which achieve complex functionality analogous to chemical signaling in biological organisms, and possibly with information processing capabilities far beyond. Light enables 
massive interconnectivity with no need for timemultiplexing schemes that have limited CMOS systems [1], [2]. For communication and signal propagation, the role of the white matter in the brain is played by waveguides, fiber optics, and free-space chip-to-chip couplers. By employing superconducting electronics, we can achieve zero static power dissipation and extraordinary device efficiencies as well as the utilization of Josephson junction (JJ) circuits including single-flux-quantum (SFQ) devices. The combination of efficient faint-light sources and superconducting-nanowire single-photon detectors interacting in an integrated-photonics environment enables neuronal operation with excellent energy efficiency, enormous intra- and inter-chip communication bandwidth, light-speed-limited latency, compact footprint, and relatively simple fabrication. Device models show the optoelectronic hardware platform can achieve $20 \mathrm{aJ} /$ synapse event, an improvement of $10^{6}$ over recent CMOS systems [1].

Memory can be implemented via several means including temporally fixed, synaptic weight variation via the actuation of locally suspended waveguides, or through the use of magnetic JJs. The suspended waveguides are reconfigurable on a time scale of $1 \mu \mathrm{s}$. None of these approaches draw power in the steady state.

Other approaches that leverage phenomena unique to optics for neuromorphic computing (NC) [3], [4], [5] have employed optical devices such as lasers and integrated microresonators. Laser cavities with strong light-matter interaction can be leveraged to realize complex nonlinear dynamics which can emulate the behavior of neurons [3], [4], [6]. The frequency selectivity of integrated ring resonators can be used to achieve synaptic weights [5]. Optical neural networks [5], [7] and spiking neurons [8], [9], [4] based on these effects have been proposed and demonstrated. The distinction of the proposed SOEN platform is that it operates in the few-photon regime with compact, energy-efficient components, enabling massive scalability.

\section{OptoelectroniC NEURONAL CIRCUITS}

A neuron based on photonic signals requires both a source and detector of photons. Here we focus on superconducting nanowire single photon detectors (SNSPDs) due to their high efficiency [10] (> 90\%), simple waveguide integration [11], compact size, and high speed. While operation at cryogenic temperatures imparts a fixed energy cost, the energy cost per operation is significantly decreased by allowing integration with superconducting electronics. Additionally, low-temperature operation also allows certain LED designs that are not possible at room temperature, as will be discussed in Sec. II-B.

\section{A. Thresholding single-photon detector array}

An SNSPD-based circuit which achieves an integrate-and-fire response with a given threshold photon number is shown in Fig. 2(a). The circuit has two components. On the left is a parallel array of nanowires, referred to as a parallel nanowire detector (PND) [12]. To achieve integrate-and-fire functionality, the PND is in parallel with an LED. The thresholding mechanism is explained pictorially in Fig. 2(b)-(e). In the steady state, the PND is superconducting and has zero resistance. The semiconducting LED has finite resistance, and therefore all current from the source $I_{1}$ flows through the PND. When a sufficient number of nanowires in the PND has been driven to the normal state by the absorption of photons, the critical current of the array is exceeded, the array becomes resistive, and current is diverted to the LED. This diversion of current and the subsequent production of light via carrier recombination constitutes the firing event. The LED fires with a step response, meaning that the LED output is independent of the exact number of photons absorbed and only depends on whether or not the threshold has been exceeded. The minimum duration of a spike event is determined by the emitter lifetime. The integration time of the neuron can be engineered to be within the range of a few hundred ps up to infinity.

Introducing an amplifier into the circuit shown in Fig. 2(a) allows decoupling of the firing threshold and LED gain. This can be achieved with an nTron, a three terminal supercurrent amplifier [13]. When the current in the gate exceeds the critical current, the path from the source to drain is driven normal, 


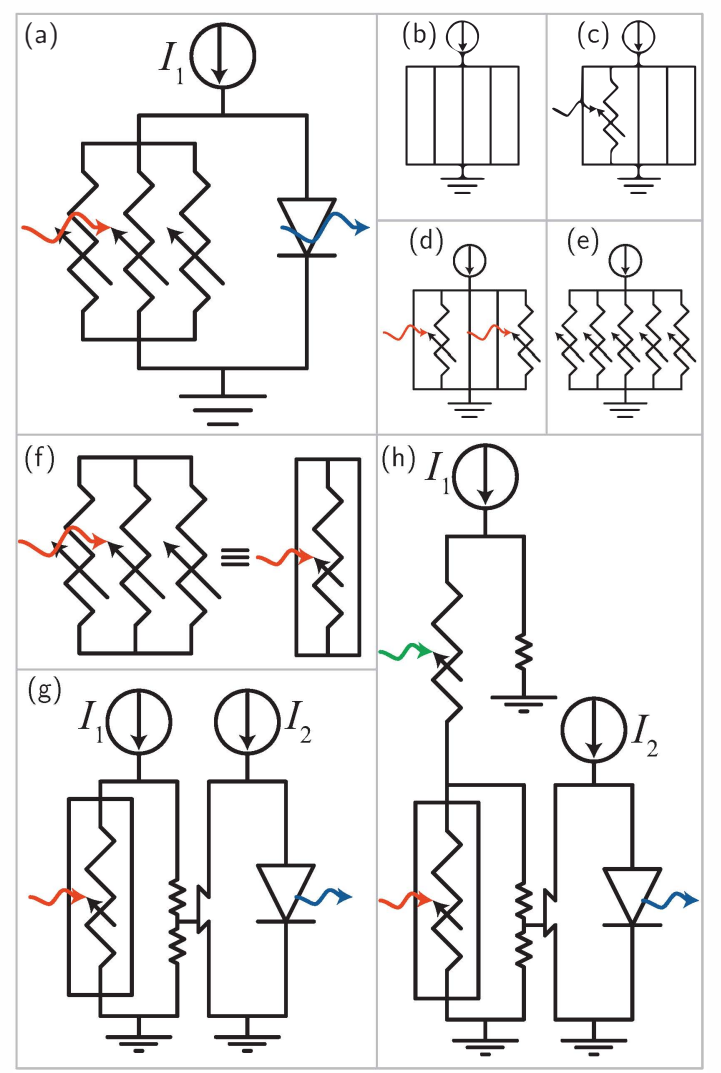

Fig. 2. (a) PND circuit. (b) A PND with all wires superconducting (c) A PND where one of the wires has been driven normal by absorption of a single photon, redirecting the current through the other three. (d) A PND with two normal wires by absorption of two photons (e) A PND with all wires driven normal by exceeding the critical current. An LED in parallel with this PND will now receive current, causing a firing event.(f) Definition of the circuit symbol representing the PND. (g) Neuron with nTron amplifier. (h) Neuron with inhibitory and excitatory connections.

diverting the bias current to the parallel load. This device has been used to drive loads of tens of $\mathrm{k} \Omega \mathrm{s}$, making it suitable for this application.

In Fig. 2 (f) we introduce a symbol which defines the PND, and in Fig. 2(g) we show a variation on the circuit of Fig. 2(a), but instead of driving the same current $I_{1}$ through the LED after firing, this circuit utilizes an nTron current amplifier to provide gain to the light emitter.

Most neuronal connections provide feedforward excitation, wherein an action potential produced by upstream neurons increases the probability of action potentials being produced by downstream neurons. Biological systems also exhibit connections wherein the firing of certain upstream neurons suppresses the probability of firing events by certain downstream neurons. Figure 2(h) shows a configuration which achieves this. The lower portion of the circuit is identical to that of Fig. $2(\mathrm{~g})$, but the current $I_{1}$ feeding the receiver first passes through a preliminary SNSPD. Absorption of photons in this region of the circuit reduces the current through the primary receiver, increasing the threshold photon number. In Fig. 2(h) the inputs to the two receivers are drawn with different colors, emphasizing the possibility that integrated photonic filters placed before the neuron could be employed to route different frequencies to the two receivers.

We refer to the spiking neurons of Fig. 2 as single-photon optoelectronic neurons (SPONs).

\section{B. Electrically-injected light source}

For the application at hand, massive scaling is a priority, and this requires photonic-electronic process integration. A single source with 100\% efficiency is less desirable than the ability to scale to millions (and eventually billions) of sources with $1 \%$ efficiency each. The device we envision consists of a $p-i-n$ junction created in a ridge waveguide. To minimize absorption in other regions of the photonic circuits, the ability to lithographically control the location of emitters is crucial.

One option is to implement these devices on a $\mathrm{GaAs}$ or InP substrate. A III-V platform has the advantage of high efficiency light sources. However, working on III-V substrates is more expensive and forbidden in CMOS foundries. Hybrid III-V/silicon integration such as direct hetero-epitaxial growth is also an option. While great progress in this field has been made [14], [15], [16], [17], [18], additional effort is needed to achieve the waveguide-integrated sources required for this NC system.

A commonly overlooked light source that may prove promising for this application is emissive centers in Si [19], [20]. These do not operate at room temperature, but the SNSPDs utilized here already require cryogenic temperatures. It is ap- 
pealing that many emissive centers can be easily fabricated in a CMOS-compatible process via ion implantation and annealing [19], [20], [21]. With co-implantation of multiple impurities it is possible to add additional (color) degrees of freedom to the NC platform. Similarly, on a III-V platform we could take advantage of inhomogeneous broadening of the quantum dot spectrum and tuning of dot size.

The NC platform proposed here is not tied to any one of these light sources. For the calculations presented here, we assume LEDs with $1 \%$ efficiency at $1.22 \mu \mathrm{m}$ in a waveguiding medium with index of 3.52 with a cladding of 1.46 above and below.

\section{Connectivity}

Of central importance to the implementation of the proposed NC platform is the network of waveguides which connect the processing units. Optical waveguides offer the possibility for improved performance over electrical connections by allowing individual neurons to integrate signals from many sources without the need for time-multiplexing. Due to the additional energy cost associated with the capacitance of additional wires, electrical neurons must utilize shared wires. Voltage pulses from different neurons on the same bus will interact. To prevent this, pulses must be delayed in time.

In the hardware platform proposed here, each neuron will have a waveguide exiting the LED and leading to many branching waveguides, which we liken to the axon and its arbor, and another set of integrating waveguides combining signals received from upstream neurons, which we liken to the dendritic arbor, as shown schematically in Fig. 1. Connections between these input and output waveguides act as synapses in this network.

\section{A. The dendritic arbor}

At each neuron, the device must be designed to combine the modes from a large number of waveguides on a receiver with low loss. Here we propose one means for accomplishing this, as shown in Fig. 3.

In this design, referred to as the stingray SPON, the input waveguides are directly combined on a landing pad housing the PND array. Figure 3(b)

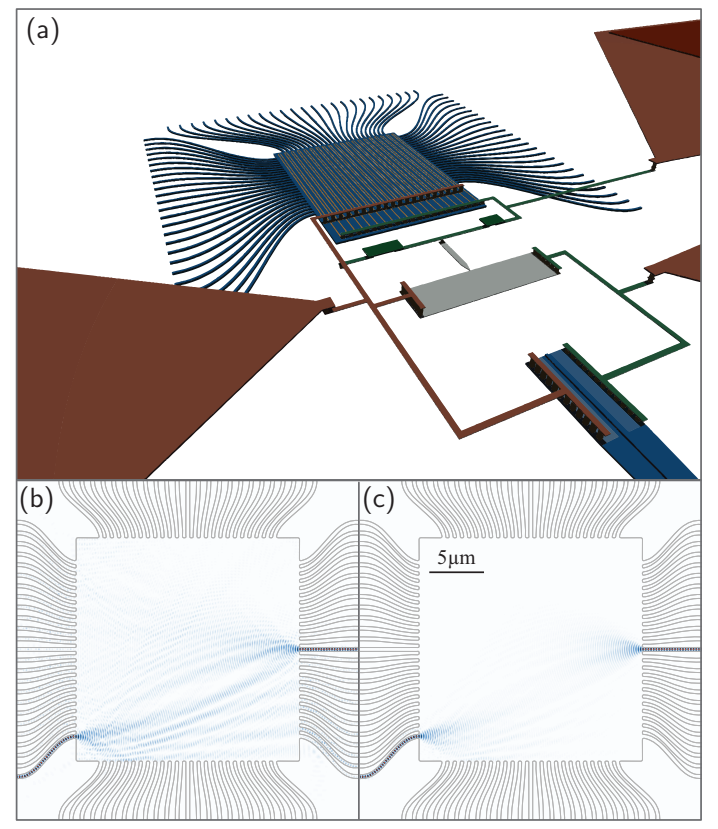

Fig. 3. (a) Overview of the stingray neuron. (b) FDTD simulation of the dendritic arbor for the stingray neuron with no SNSPDs present to absorb the light and (c) with SNSPDs present.

and (c) show 2D finite-difference time-domain simulations of the structure. Figure 3(b) shows the propagation of light into the receiver body in the absence of absorbing nanowires, while Fig. 3(c) shows the propagation through the absorbing nanowire array. Here, 100 waveguides terminate on a receiver body with less than $0.2 \mathrm{~dB}$ insertion loss from any port, with the outer-most ports giving the most loss [bottom illuminated port in Fig. 3(b) and (c)], and the inner-most (central) ports achieving near zero insertion loss. In this context, insertion loss refers to light entering and leaving the simulation without being absorbed in the nanowire array. The entire receiver of Fig. 3(c) occupies $30 \mu \mathrm{m} \times 30 \mu \mathrm{m}$. A design with 204 input waveguides and less than 1 $\mathrm{dB}$ insertion loss with a footprint of $60 \mu \mathrm{m} \times 60 \mu \mathrm{m}$ has also been found. For larger numbers of inputs, the simulations become cumbersome. Yet continued scaling is possible. 


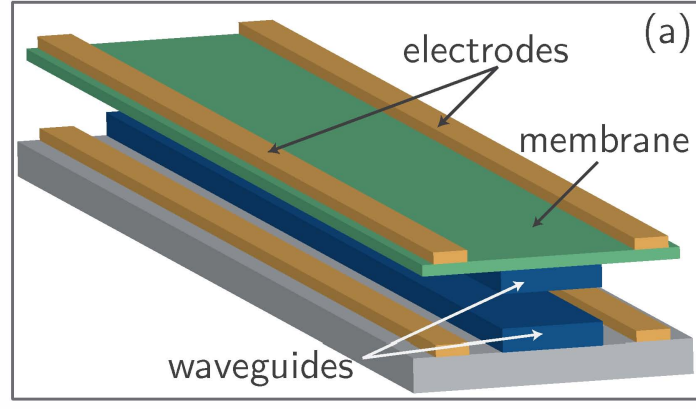

Fig. 4. Interlayer waveguide coupler with electro-mechanically tunable coupling.

\section{B. The axon and its arborization}

The output waveguide from a unit's LED (axon) must split into as many branches as there are connections to be made. Such power splitters can be made with a small footprint and low loss. It is straightforward to generalize such power splitters into the third dimension with multilayer photonics, and such an implementation would enable thousands of synapses with a volume of 10 $\mu \mathrm{m}^{3}$ /synapse.

\section{Learning, reconfiguration, and plasticity}

An important aspect of any NC system is the ability to establish the strength of interaction between connected units. These connection strengths, often referred to as the weight matrix, are important for memory and learning. This weight matrix determines how much of the light from the firing of a particular neuron is coupled into any other neuron, analogous to the synaptic strength between two neurons in a biological system.

Varying the strength of interaction between neurons is challenging at cryogenic temperatures, where modulators that rely on either the thermooptic effect or free carrier injection are ineffective, and electro-optic switches require too much space for this application. We propose the employment of electro-mechanically actuated waveguide couplers, schematically depicted in Fig. 4. These waveguides can be coupled either vertically (as shown in Fig. 4) or laterally. This distance can be controlled electromechanically, and anywhere from $0 \%$ to $100 \%$ of the light can be coupled from one waveguide to the other. The minimum coupling would be set in hardware, as the gap at $0 \mathrm{~V}$ is the maximum. Any applied voltage (positive or negative) produces an attractive force between the two waveguides. Activity in the circuit can induce voltage between the waveguides, and increase the strength of the synapse. Such couplers have recently been demonstrated [22] in a highly scaled configuration. In ref. [22], 4096 such switches were operated with $>60$ $\mathrm{dB}$ extinction ratio.

For highly scaled implementations emulating the behavior of biological organisms, it is important that each synapse be as small as possible to enable massive scaling, but it is also important that voltages be modest, as we would like activity in the circuits to be capable of reconfiguring the synapses. In particular, we would like firing events from upstream neurons followed closely by firing events by downstream neurons to place charge on this MEMS capacitor (waveguide coupler), and thereby increase the synaptic strength. This coordinated charging of the membrane would accomplish spiketiming-dependent plasticity. The storage of charge on a capacitor required for this device operation is very similar to dynamic random access memory (DRAM), which is a mature technology. While implementing spike-timing-dependent DRAM with suspended waveguide membranes presents a technical challenge, it offers a promising means to implement truly neuromorphic learning within this optoelectronic platform.

\section{NETWORKS AND SCALING}

We have now discussed neural circuits based on optical signaling. We have discussed means to connect these optical and electrical signals in a timevarying manner with event-based plasticity. We now consider networks of these neurons, which we refer to as superconducting optoelectronic networks (SOENS). To assess the scaling of SOENs, we consider both the energy and spatial consumption.

\section{A. Energy consumption}

Considering the PND circuit of Fig. 2(g), we decompose the energy consumption into three con- 

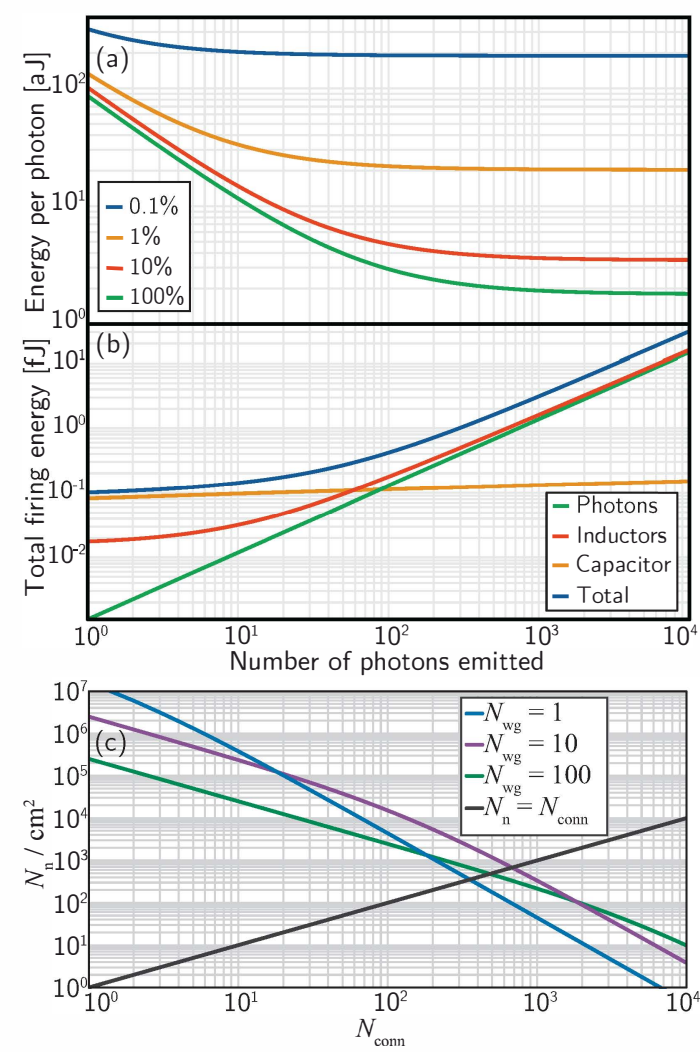

Fig. 5. (a) Energy required to generate a single photon versus number of photons emitted for four different LED efficiencies. (b) Contributions to total energy consumption for a $10 \%$ efficient LED. (c) Number of neurons per $\mathrm{cm}^{2}$ versus number of connections per neuron.

tributions: the inductive energy stored in the supercurrents flowing through the circuit elements, the capacitive energy associated with the LED, and the energy required to produce the photons, associated with the current through the LED. In this model, we assume one inductor $L_{\text {SNSPD }}$ in the PND array for each photon, as well as a series inductance to achieve the desired temporal response. We assume each element of the PND is 500 squares, while the entire receiver array is in series with 5,000 squares of inductance. At low photon numbers the energy consumption from inductance is dominated by the series inductance, but for higher numbers it is dominated by the PND array and grows linearly. The energy required for photon production is calculated simply as $E_{g} n_{\nu} / \eta$, where $E_{g}$ is the band gap of $\mathrm{Si}$, $n_{v}$ is the number of photons created, and $\eta$ is the efficiency. We assume a superconducting material with $400 \mathrm{pH} / \square$ (such as WSi), and a parallel-plate capacitive model for the LED.

In Fig. 5(a) we plot the total energy per photon as a function of the number of photons emitted for four values of LED efficiency. We find that with a unityefficiency LED, the energy per photon could be as low as 2 aJ when larger photon numbers are created. This is still an order of magnitude greater than the $0.16 \mathrm{aJ}$ stored in the $h v$ of the light quantum itself (assuming $\lambda=1.22 \mu \mathrm{m}$ ), with the extra energy going to supplying current to the inductors and charge to the capacitor. The figure reveals that producing LEDs with efficiency above $10 \%$ has only a modest benefit, as the contribution to energy consumption from inductance will become the limiting factor. While a $100 \%$ efficient LED may not be realized, even a $1 \%$ efficient LED leads to $20 \mathrm{aJ} /$ photon, a profoundly small number compared to the $26 \mathrm{pJ}$ of a CMOS neuron [1].

In Fig. 5(b) we show the contribution to the total energy from the various circuit elements for the case of a $10 \%$ efficient LED. This efficiency is chosen for this plot because it is the value at which the contributions from inductance and photon production are nearly equal for photon numbers near or above 100. For low photon numbers, the dominant contribution is in charging the LED capacitor. Due to the highly nonlinear LED currentvoltage relationship, a small increase in the voltage across the LED leads to a large gain in current. The capacitive energy is nearly constant across the range of photon numbers considered here, and for larger photon numbers it makes a negligible contribution.

We believe an LED with $1 \%$ system efficiency is realistic in a nanophotonic environment at cryogenic temperature and with faint light levels desired. Therefore, we use $20 \mathrm{aJ} /$ photon as a representative number for what this NC platform can hope to achieve. We use the energy per photon as the energy per firing event per synapse (commonly referred to as the energy per synapse event), because the goal of the system is to produce neurons which threshold 
on a number of photons roughly equivalent to the number of connections made by the neuron. Therefore, the energy per synapse event is calculated as the total energy of the firing event divided by the number of connections. The TrueNorth chip, designed by IBM and implemented in Samsung's 28 nm CMOS process, achieved $26 \mathrm{pJ} /$ synapse event [1]. Thus, the use of superconducting electronics and faint light signals provides a factor of $10^{6}$ improvement in energy efficiency. From the second law of thermodynamics, to keep a system at $2 \mathrm{~K}$, the cooling power required is $150 \mathrm{~W} / \mathrm{W}$. Assuming a $15 \%$ efficient cooling system, this gives an estimate of $1 \mathrm{~kW} / \mathrm{W}$. Multiplying our conservative estimate of $20 \mathrm{aJ} /$ synapse event by this factor of $10^{3}$, we still have an efficiency which is 1,000 times better than CMOS. Further, while transistor technologies inevitably leak current, SPON devices draw no power in the steady state and can be DC biased without loss using JJs [23].

\section{B. Spatial scaling}

To assess the size of SPON systems, we consider a model of a feedforward multi-layer perceptron (MLP) wherein every neuron in a given MLP layer is connected to every neuron in the next MLP layer with a variable-weight connection. We consider the number of neurons in an area of one $\mathrm{cm}^{2}$ versus the number of connections per neuron, $N_{\text {conn. }}$. Figure 5(c) shows the results of this spatial model for various numbers of vertically stacked waveguiding planes, $N_{\mathrm{wg}}$. If $N_{\text {conn }}=10$ is sufficient for a given application, we can fit 400,000 neurons $/ \mathrm{cm}^{2}$. Due to the size of interlayer couplers, this is achieved more compactly with $N_{\mathrm{wg}}=1$ than with $N_{\mathrm{wg}}=10$. For $N_{\text {conn }}$ in the range of $100-1,000$, it becomes advantageous to utilize $N_{\mathrm{wg}}=10$. For $N_{\text {conn }}=100$, over 10,000 neurons will fit within a $\mathrm{cm}^{2}$, and for $N_{\text {conn }}=1,000,300$ neurons will fit within a $\mathrm{cm}^{2}$. It does not become advantageous to use $N_{\mathrm{wg}}=100$ until $N_{\text {conn }}=2,000$.

The range of $N_{\text {conn }}=100-1,000$ is promising and technologically consequential. As is the case for scaling CMOS NC platforms, utilization of die tiling will play a crucial role for this technology [1]. Die can be tiled in 2D with several types of connectivity to adjacent die including electrical, SFQ, and photonic communication over inter-die bridge waveguides. Additionally, tiling in the third dimension is possible with the usual bump bonding approach for electrical connectivity as well as with free-space optical signals sent from one chip using vertical grating couplers and received by a chip above or below using SNSPD arrays [24]. From Fig. 5(c) we find that 700 neurons with 700 connections per neuron can fit on a $1 \mathrm{~cm} \times 1 \mathrm{~cm}$ die if 10 waveguiding planes are utilized. We envision tiling a $100 \times 100$ array of these die in a plane to build a system of $7 \times 10^{6}$ neurons. This sheet will be $\sim 1$ $\mathrm{mm}$ thick, so to form a cube one meter on a side we envision tiling 1,000 vertical sheet. The system would then comprise $10^{7}$ die and $7 \times 10^{10}$ neurons, or roughly $10 \%$ the number in the human brain.

Such a device will consume $2 \times 10^{-17} \mathrm{~J} /$ synapse event, and with 700 connections, each firing event consists of 700 synapse events. Information processing in NC systems requires sparse event rates, so for the SOEN hardware wherein $20 \mathrm{MHz}$ is achievable based on device limitations, $20 \mathrm{kHz}$ represents a sparse rate. For the system under consideration, this gives a total power consumption of $2 \mathrm{~W}$. This equates to $5 \times 10^{16}$ synapse events per second per watt. The system must be kept around $2 \mathrm{~K}$, so an additional $1 \mathrm{~kW} / \mathrm{W}$ must be supplied as cooling power, as discussed in Sec. IV-A. While this does not affect the power density (which ultimately limits scaling), if we include this additional power in the calculation we find that we achieve $5 \times 10^{13}$ synapse events per second per watt.

For comparison, we assume a human being uses $100 \mathrm{~W}$ for $10^{11}$ neurons with $7 \times 10^{3}$ synapses per neuron firing at $1 \mathrm{~Hz}$. This equates to $7 \times 10^{12}$ synapse events per second per watt. Even with the $1 \mathrm{~kW} / \mathrm{W}$ cooling power of the cryostat, we find that the number of synapse events per second per watt of the SOEN system exceeds that of the brain.

Importantly, because signaling occurs predominantly in the optical domain, firing events can be directly imaged with a camera. For massively scaled systems, this becomes a powerful metrological tool. Such a measurement technique can be used to monitor device and system performance across 
spatial and temporal scales in a manner analogous to functional magnetic resonance imaging.

\section{SUMMARY}

We have described a hardware platform combining superconducting electronics and semiconducting faint photon sources to operate as a massively interconnected information processing system. The SOEN platform consists of neurons that exhibit complex signaling and efficient access to photonic degrees of freedom such as frequency, polarization, mode index, intensity, and coherence, in analogy to the complex signaling mechanisms in the brain. The proposed networks of connections, based on reconfigurable waveguides, offer advantages over electronic connections in terms of speed, connectivity, and energy efficiency.

\section{REFERENCES}

[1] P. Merolla, J. Arthur, R. Alvarez-Icaza, A. Cassidy, J. Sawada, F. Akopyan, B. Jackson, N. Imam, C. Guo, Y. Nakamura, B. Brezzo, I. Vo, S. Esser, R. Appuswamy, B. Taba, A. Amir, M. Flickner, W. Risk, R. Manohar, and D. Modha, "A million spiking-neuron integrated circuit with scalable communication network and interface," Science, vol. 345, p. 668, 2014.

[2] J. Hasler and B. Marr, "Finding a roadmap to achieve large neuromorphic hardware systems," Front. Neurosci., vol. 7, p. 118, 2013.

[3] W. Coomans, L. Gelens, S. Beri, J. Danckaert, and G. V. der Sande, "Solitary and coupled semiconductor ring lasers as optical spiking neurons," Phys. Rev. E, vol. 84, p. 036209, 2011.

[4] M. Nahmias, B. Shastri, A. Tait, and P. Prucnal, "A leaky integrate-and-fire laser neuron for ultrafast cognitive computing," IEEE J. Sel. Top. Quant. Electron., vol. 19, p. 1800212, 2013.

[5] A. Tait, M. Nahmias, Y. Tian, B. Shastri, and P. Prucnal, Nanophotonic Information Physics, M. Naruse, Ed. Springer.

[6] B. Shastri, M. Nahmias, A. Tait, A. Rodriguez, B. Wu, and P. Prucnal, "Spike processing with a graphene excitable laser," Nature Sci. Rep., vol. 6, p. 19126, 2016.

[7] E. C. Mos, J. J. H. B. Schleipen, and H. de Waardt, "Optical-mode neural network by use of the nonlinear response of a laser diode to external optical feedback," Applied Optics, vol. 36, no. 26, p. 6654, sep 1997.

[8] K. S. Kravtsov, M. P. Fok, P. R. Prucnal, and D. Rosenbluth, "Ultrafast all-optical implementation of a leaky integrate-and-fire neuron.” Optics express, vol. 19, no. 3, pp. 2133-47, jan 2011.

[9] A. Hurtado, K. Schires, I. D. Henning, and M. J. Adams, "Investigation of vertical cavity surface emitting laser dynamics for neuromorphic photonic systems," Applied Physics Letters, vol. 100, no. 10, p. 103703, mar 2012.
[10] F. Marsili, V. Verma, J. Stern, S. Harrington, A. Lita, T. Gerrits, I. Vayshnker, B. Baek, M. Shaw, R. Mirin, and S. Nam, "Detecting single infrared photons with 93p. 210, 2013.

[11] D. Sahin, A. Gaggero, J.-W. Weber, I. Agafonov, M. Verheijen, F. Mattioli, J. Beetz, M. Kamp, S. Höfling, M. van de Sanden, R. Leoni, , and A. Fiore, "Waveguide nanowire superconducting single-photon detectors fabricated on gaas and the study of their optical properties," IEEE J. Sel. Top. Quant. Electron., vol. 21, p. 3800210 , 2015.

[12] F. Marsili, D. Bitauld, A. Gaggero, S. Jahanmirinejad, R. Leoni, F. Mattioli, and A. Fiore, "Physics and application of photon number resolving detectors based on superconducting parallel nanowires," New J. Phys., vol. 11, p. 045022, 2009.

[13] A. McCaughan and K. Berggren, "A superconductingnanowire three-terminal electrothermal device," Nanolet ters, vol. 14, p. 5748, 2014.

[14] Z. Yuan, B. Kardynal, R. Stevenson, A. Shields, C. Lobo, K. Cooper, N. Beattie, D. Ritchie, and M. Pepper, "Electrically driven single-photon source," Science, vol. 295, p. 102, 2002.

[15] A. Shields, "Semiconductor quantum light sources," Nature Photonics, vol. 1, p. 215, 2007.

[16] A. Liu, C. Zhang, J. Norman, A. Snyder, D. Lubyshev, J. Fastenau, A. Liu, A. Gossard, and J. E. Bowers, "High performance continuous wave $1.3 \mu \mathrm{m}$ quantum dot lasers on silicon," Appl. Phys. Lett., vol. 104, p. 041104, 2014.

[17] Y. Wan, Q. Li, Y. Geng, B. Shi, and K. Lau, "Inas/gaas quantum dots on gaas-on-v-grooved-si substrate with high optical quality in the $1.3 \mu \mathrm{m}$ band," Appl. Phys. Lett., vol. 107, p. 081106, 2015.

[18] H. Schmid, M. Borg, K. Moselund, L. Gignac, C. Breslin, J. Bruley, D. Cutaia, and H. Riel, "Template-assisted selective epitasy of iii-v nanoscale devices for co-planar heterogeneous integration with si," Appl. Phys. Lett., vol. 106, p. 233101, 2015.

[19] G. Davies, "The optical properties of luminescence centres in silicon,” Physics Reports, vol. 176, pp. 83-188, 1989.

[20] J. Shainline and J. Xu, "Silicon as an emissive optical medium," Laser and Photonics Reviews, vol. 1, p. 334, 2007.

[21] H. Sumikura, E. Kuramochi, H. Taniyama, and M. Notomi, "Ultrafast spontaneous emission of copper-doped silicon enhanced by an optical nanocavity," Sci. Rep., vol. 4, p. 5040, 2014

[22] T. Seok, N. Quack, S. Han, R. Muller, and M. Wu, "Largescale broadband digital silicon photonic switches with vertical adiabatic couplers," Optica, vol. 3, p. 64, 2016.

[23] D. Kirichenko, S. Sarwana, and A. Kirichenko, "Zero static power dissipation biasing of rsfq circuits," IEEE Trans. Appl. Supercond., vol. 21, p. 776, 2011.

[24] M. Allman, V. Verma, M. Stevens, T. Gerrits, R. Horansky, A. Lita, F. Marsili, A. Beyer, M. Shaw, D. Kumor, R. Mirin, and S. Nam, "A near-infrared 64-pixel superconducting nanowire single photon detector array with integrated multiplexed readout," Appl. Phys. Lett, vol. 106, p. 192601, 2015. 